\title{
NUMERICAL ANALYSIS OF HEAT AND MASS TRANSFER ALONG A STRETCHING WEDGE SURFACE
}

\author{
M. Ali ${ }^{1}$, M. A. Alim², R. Nasrin' ${ }^{3}$ M. S. Alam ${ }^{4}$ \\ ${ }^{1}$ Department of Mathematics, Chittagong University of Engineering and Technology, Assistant Professor, \\ E-mail:ali.mehidi93@gmail.com \\ ${ }^{2}$ Department of Mathematics, Bangladesh University of Engineering and Technology, Professor, \\ E-mail:a0alim@gmail.com \\ ${ }^{3}$ Department of Mathematics, Bangladesh University of Engineering and Technology, Associate Professor, \\ E-mail:raity11@gmail.com \\ ${ }^{4}$ Department of Mathematics, Chittagong University of Engineering and Technology, Associate Professor, \\ E-mail:shahalammaths@ gmail.com
}

\section{Abstract:}

In this work, the effects of dimensionless parameters on velocity field, thermal field and nanoparticle concentration field have been studied. In this regard, the governing partial differential equations are transformed into ordinary differential equations by using similarity transformations. These transformed equations are then solved numerically using the function bvp $4 c$ of MATLAB for different values of the parameters. The values of magnetic parameter have been considered as $0.0,0.5,1.0$ \& 1.5 , stretching ratio as 0.0, 0.3, $0.5 \& 0.7$, Brownian motion as 0.0, 0.1, 0.15, 0.20, 0.3, 0.5, 0.8 \& 1.5, and thermophoresis as 0.0, 0.05, 0.1, 0.14, 0.3, $0.6 \& 1.0$. The Prandt number and Lewis number are taken as $(1.0,3.0 \& 6.0)$ and $(1.0,2.0,3.0 \& 5.0)$ respectively. The results indicate that the velocity field increases for the increase in values of pressure gradient parameter, magnetic parameter and stretching ratio parameter. The temperature field decreases for the increase in values of stretching ratio parameter, Brownian motion parameter and Prandt number but reverse result arises for the increase in values of thermophoresis parameter. The nanoparticle concentration field decreases for the increase in values of pressure gradient parameter, Brownian motion parameter and Lewis number whereas it increases as thermophoresis parameter increases. Finally, for validity and accuracy, the present results has compared with previously published work and found to be in good agreement.

Keywords: MHD, nanofluid, wedge flow, pressure gradient.

$\begin{array}{llll}\text { NOMENCLATURE } & \text { Le } & \text { Lewis number } \\ u, v & \text { velocity components } & N b & \text { Brownian motion } \\ f & \text { dimensionles velocity stream function } & N t & \text { thermophoresis parameter } \\ f^{\prime} & \text { dimensionless velocity } & M & \text { magnetic parameter } \\ x, y & \text { distance from the surface } & \text { Greek } & \text { symbols } \\ M H D & \text { magnetohydrodynamic } & \alpha_{b} & \text { dynamic viscosity } \\ B_{0} & \text { magnetic field } & v_{b} & \text { kinematic viscosity } \\ m & \text { pressure gradient related to wedge angle } & \rho_{b} & \text { density of the base fluid } \\ T & \text { temperature of the fluid } & \Omega & \text { total wedge angle } \\ T_{w} & \text { temperature of the surface } & \beta & \text { wedge angle } \\ T_{\infty} & \text { temperature of the free stream fluid } & \lambda & \text { stretching ratio } \\ C & \text { concentration of the fluid } & \eta & \text { dimensionless variable } \\ C_{w} & \text { concentration of the boundary } & \tau & \text { ratio of heat capacity } \\ C_{\infty} & \text { concentration of the free stream fluid } & \sigma & \text { electrical conductivity } \\ D_{B} & \text { Brownian diffusion coefficient } & K & \text { thermal conductivity }\end{array}$




$\begin{array}{llll}D_{T} & \text { thermophoresis diffusion coefficient } & \Psi & \text { stream function } \\ \mathrm{Nu} & \text { Nusselt number } & \theta & \text { dimensionless temperature } \\ \mathrm{Sh} & \text { Sherwood number } & \varphi & \text { dimensionless concentration } \\ \mathrm{Pr} & \text { Prandtl number } & & \end{array}$

\section{Introduction}

The main application of the boundary layer theory is the calculation of skin friction drag that acts on a body, which is moving in a fluid, such as the drag of a flat plate, an airplane wing, a turbine blade, or a complete ship. In case of convective heating or cooling purpose, such as, cooling of an engine and cooling of a hot plate in case of an external flow condition, a fluid boundary layer is formed that gives rise to thermal boundary layer. Besides, moving or stretching surface is important in many engineering processes such as production of polymeric sheets, paper production, wire drawing, insulating materials, drawing of plastic films and fine-fiber mats etc. So on the basis of the Prandtl boundary layer theory, Falkner-Skan developed a model that was not parallel to the fluid flow known as wedge flow.

Adekeye et al. (2017) identified that a strong flow circulation arises at particular value of Grashof number and heat transfer rate is significant at certain interval of inclination. Ahmed et al. (2014) observed that the skin friction coefficient decreases as the Reynolds number and the suction/injection parameter increases, while the local Nusselt number increases as the Reynolds number and the suction/injection parameter increases. Further Ahmed et al. (2014) explained that the local and average Nusselt numbers at the hot and cold sidewalls increase with increasing the radiation parameter. From the other side, the role of viscous dissipation parameter is to reduce the local and average Nusselt numbers at the hot left wall, while it improves them at the cold right wall. Ashwini et al. (2015) obtained that the dual solutions exist up to a certain value of unsteady parameter beyond which, the boundary layer separates from the surface. Besides the magnetic parameter delays the boundary layer separation because the flow is accelerated. Bharathi (2017) observed that the velocity is higher for Pseudoplastic fluids and temperature is higher for Dilatant fluids.

Again, Choi (1995) was the first who discussed the concept of nanofluid, which refers to the dispersions of nanoparticles in the base fluids such as water, ethylene glycol, and propylene glycol. Later, Buongiorno (2006) had first examined the reasons behind the enhancement in heat transfer rate for nanofluid and he observed that the Brownian motion and thermophoresis are the main causes to enhance heat transfer rate. Chand et al. (2015) indicated that the Prandtl and Darcy numbers have a destabilizing effect while the Lewis number and modified diffusivity ratio have a stabilizing effect for the stationary convection. Falana et al. (2016) discussed that the temperature increases with an increase in the thermophoresis parameter or Brownian motion parameter or stretching parameter.

Hayat et al. (2011) discussed the Falkner- Skan flow in case of power - law fluid with mixed convection. Hussein et al. (2014) shown that the solid volume fraction has a significant influence on stream function and heat transfer, depending on the value of Hartmann and Rayleigh numbers. Khan and Pop (2013) noticed that the reduced Nusselt number is a decreasing function of each dimensionless number, while the reduced Sherwood number is an increasing function of higher Prandtl number and a decreasing function of lower Prandtl number for each Lewis number, Brownian motion and thermophoresis parameters. Haile (2015) analyzed the effects of various parameters on boundary layer nanofluid flow past a moving surface and obtained that the fluid flow and heat transfer are influenced by magnetic parameter, Brownian motion and thermophoresis parameter. Shaw et al. (2016) proved that the existence and uniqueness of the solutions depends on the slip parameters, and that the region of existence of the dual solution increases with the slip parameters. Srinivasacharya et al. (2015) performed that the magnetic parameter, Falkner-Skan power-law parameter and the volume fraction parameter are the key parameter for heat and mass transfer rate. Khan et al. (2017) examined that the velocity field increases in both stretching and shrinking wedges for pressure gradient parameter and magnetic parameter. Kandasamy (2015) discussed the radiative heat transfer on nanofluid flow over a porous convective surface in the presence of magnetic field. Nasrin et al. $(2011,2012,2014)$ studied heat and mass transfer enhancement based on MHD effect considering different types of geometry. However, boundary layer nanofluid flow past a stretching wedge surface in the presence of magnetic field are widely studied in a comprehensive way. Yousif 
(2017) concluded that increasing the transpiration parameter, nanoparticles concentration over the plate decrease due to more fluid penetration from pores and this is the main reas on of lower thermal boundary layer caused by fewer nanoparticles over the plate. After that, Yacob et al. (2011) noticed that the rate of velocity and the rate of heat transfer at the surface are highest for copper-water nanofluid compared to the alumina-water and titaniawater nanofluid.

\section{Physical Model}

Let us consider the wedge shape surface which is moving with a velocity $u_{w}(x)$ and the free stream velocity is $U_{e}(x)$.The x-axis is measured along the surface of the wedge and the positive y-coordinate measured normal to the $\mathrm{x}$-axis in the outward direction. The temperature of the wedge wall $\mathrm{T}_{\mathrm{w}}$ and nanoparticle concentration $\mathrm{C}_{\mathrm{w}}$ are variable and the free stream temperature and nanoparticle concentration are $\mathrm{T}_{\infty}$ and $\mathrm{C}_{\infty}$ respectively far away from the boundary layer, the total angle of the wedge is $\Omega=\beta \pi$, where $\beta$ is the Hartree pressure gradient that are shown in Fig. 1.

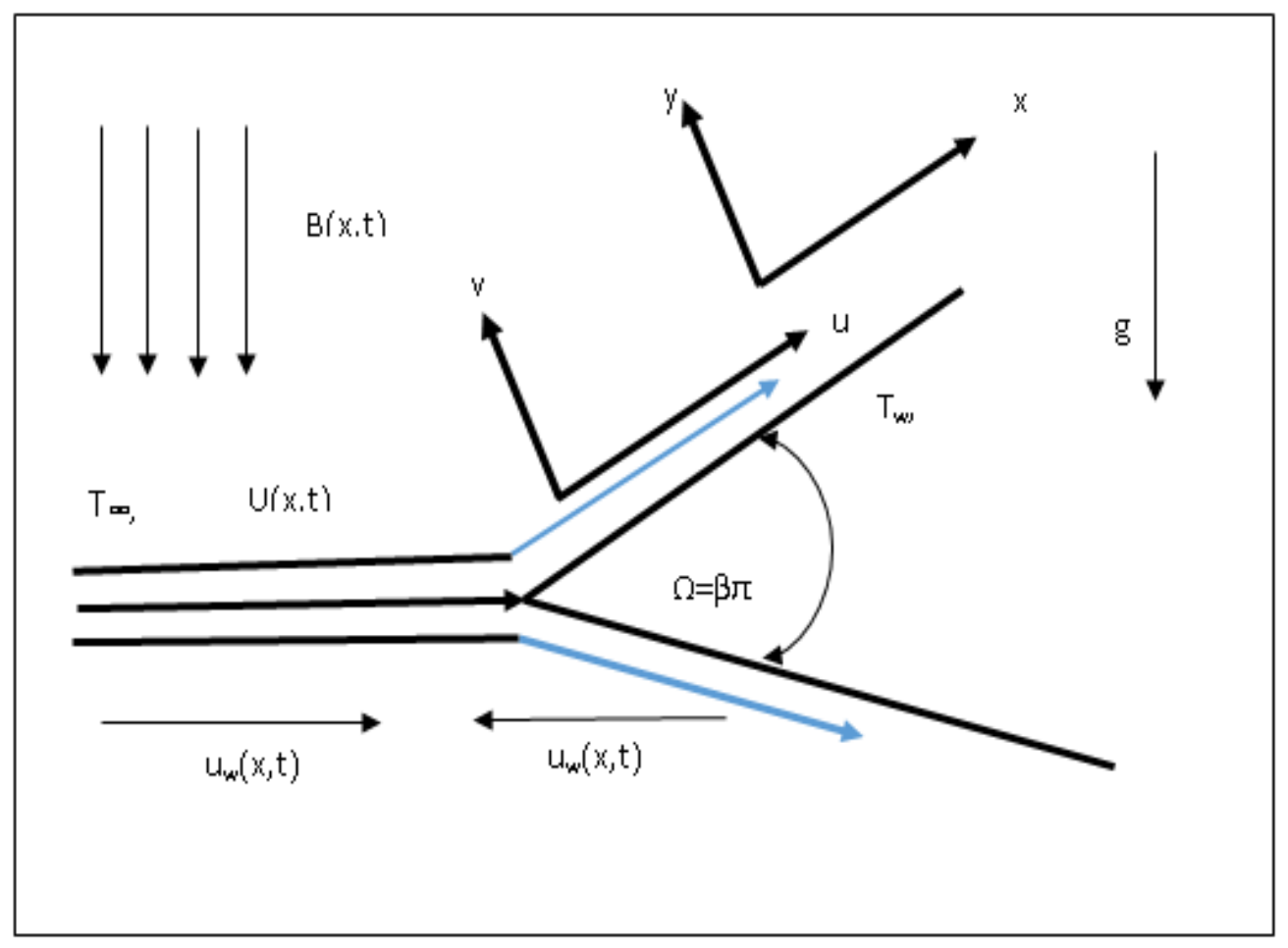

Fig. 1: A flow configeration and coordinate system

\subsection{Thermo-physical properties}

The thermo-physical properties of the nanofluid are taken from Parvin et al. (2012) and given in Table 1.

Table 1: Thermo-physical properties of water and $\mathrm{Cu}$ nanoparticle at $300 \mathrm{~K}$

\begin{tabular}{ccc}
\hline Physical Properties & Fluid phase (water) & $\mathrm{Cu}$ \\
\hline$C_{p}(\mathrm{~J} / \mathrm{kgK})$ & 4179 & 385 \\
$\rho\left(\mathrm{kg} / \mathrm{m}^{3}\right)$ & 997.0 & 8933 \\
$k\left(\mathrm{~W} / \mathrm{mK}^{2}\right.$ & 0.613 & 400 \\
$\alpha \times 10^{7}\left(\mathrm{~m}^{2} / \mathrm{s}\right)$ & 1.47 & 1163.1 \\
$\mu \times 10^{6}\left(\mathrm{Ns} / \mathrm{m}^{2}\right)$ & 855 & - \\
\hline
\end{tabular}




\section{Governing Equations and Similarity Analysis}

The governing partial differential equations for the boundary-layer flow of nanofluid for the present problem can be written as follows (Makinde et al., 2013):

Equation of continuity:

$$
\frac{\partial u}{\partial x}+\frac{\partial v}{\partial y}=0
$$

Momentum equation:

$$
u \frac{\partial u}{\partial x}+v \frac{\partial u}{\partial y}=U \frac{d U}{d x}+v_{b} \frac{\partial^{2} u}{\partial y^{2}}+\frac{\sigma B_{0}^{2}}{\rho_{b}}(U-u)
$$

Energy equation:

$$
u \frac{\partial T}{\partial x}+v \frac{\partial T}{\partial y}=\alpha_{b} \frac{\partial^{2} T}{\partial y^{2}}+\tau\left\{D_{B}\left(\frac{\partial T}{\partial y} \frac{\partial C}{\partial y}\right)+\frac{D_{T}}{T_{\infty}}\left(\frac{\partial T}{\partial y}\right)^{2}\right\}
$$

Nanoparticle concentration equation:

$$
u \frac{\partial C}{\partial x}+v \frac{\partial C}{\partial y}=D_{B} \frac{\partial^{2} C}{\partial y^{2}}+\frac{D_{T}}{T_{\infty}} \frac{\partial^{2} T}{\partial y^{2}}
$$

The above equations are subject to the following boundary conditions:

$$
\begin{aligned}
& u=u_{w}(x, t), v=0, T=T_{w}, C=C_{w} \text { at } y=0 \\
& u=U(x, t), T \rightarrow T_{\infty}, C \rightarrow C_{\infty} \text { as } y \rightarrow \infty
\end{aligned}
$$

The velocity of the wedge surface, the free stream velocity, temperature and nanoparticle concentration can be taken as follows:

$$
u_{w}(x, t)=a x^{m}, U(x, t)=b x^{m}, T_{w}-T_{\infty}=b x^{m}, C_{w}-C_{\infty}=b x^{m} .
$$

where $a$ and $\mathrm{b}$ are positive constant on which $b$ is the initial stretching rate, and the exponent $m$ is a function of the wedge angle parameter $\beta$ where the total apex angle of the wedge is $\beta \pi$ such that $\beta=\frac{2 m}{1+m}$.

To convert the governing equations into a set of ordinary differential equations, following similarity transformations are introduced:

$$
\eta=\mathrm{y} \sqrt{\frac{\mathrm{U}(1+\mathrm{m})}{2 x v}}, \psi=\sqrt{\frac{2 x v U}{(1+m)}} f(\eta), \theta(\eta)=\frac{T-T_{\infty}}{T_{w}-T_{\infty}}, \varphi(\eta)=\frac{C-C_{\infty}}{C_{w}-C_{\infty}}, u=\frac{\partial \psi}{\partial y} \quad \text { and } v=-\frac{\partial \psi}{\partial x}
$$

By applying the above similarity transformations, the partial differential Eq. (2), Eq. (3) and Eq. (4) transformed into non-dimensional, nonlinear and coupled ordinary differential equations as follows:

$$
\begin{aligned}
& f^{\prime \prime \prime}+f f^{\prime \prime}+\beta\left(1-f^{\prime 2}\right)+M\left(1-f^{\prime}\right)=0 \\
& \theta^{\prime \prime}+\operatorname{Pr}\left[f \theta^{\prime}-\beta f^{\prime} \theta+N b \theta^{\prime} \varphi^{\prime}+N t \theta^{\prime 2}\right]=0
\end{aligned}
$$




$$
\varphi^{\prime \prime}+\frac{N t}{N b} \theta^{\prime \prime}+L e\left[f \varphi^{\prime}-\beta f^{\prime} \varphi\right]=0
$$

The transformed boundary conditions are:

$$
\begin{aligned}
& f=0, f^{\prime}=\lambda, \theta=1, \phi=1 \text { at } \eta=0 \text { and } f^{\prime} \rightarrow 1, \theta=\phi \rightarrow 0 \text { as } \eta \rightarrow \infty \\
& M=\frac{2 \sigma B_{0}^{2} U x}{\rho_{f}}, \lambda=\frac{a}{b}, \operatorname{Pr}=\frac{{ }^{v} f}{\alpha_{f}}, N b=\frac{\tau D_{B}\left(C_{w}-C_{\infty}\right)}{v_{f}}, N t=\frac{\tau D_{T}\left(T_{w}-T_{\infty}\right)}{T_{\infty} v_{f}}, \beta=\frac{2 m}{1+m}, L e=\frac{v}{D_{B}}
\end{aligned}
$$

The important physical quantities of this problem are skin friction coefficient $C_{f}$, the local Nusselt number $N u$ and the local Sherwood number $S h$ which are proportional to rate of velocity, rate of temperature and rate of nanoparticle concentration respectively.

\section{Numerical Modeling}

The non-linear ordinary differential Eq. (5) - Eq. (7) with the boundary conditions has been performed by applying shooting method namely Nachtsheim and Swigert [26] iteration technique along with fourth order Runge-Kutta iteration scheme to get the numerical results. From Eq. (5) - Eq. (7) it is observed that $f$ is in third order and $\theta$ and $\varphi$ are in second order. In order to solve this system of equations using Runge-Kutta method, the solution needs seven initial conditions but we have two initial conditions in $f$ and one initial condition in each of $\theta$ and $\varphi$. The most important step of this scheme is to choose the appropriate finite value of $\eta_{\infty}$. Therefore, to determine the value of $\eta_{\infty}$, the procedure has to be started with some initial guess value and solve the boundary value problem consisting of Eq. (5) - Eq. (7). The solution process is repeated with another larger value of $\eta_{\infty}$ until two successive values of $f^{\prime \prime}(0), \theta^{\prime}(0)$ and $\phi^{\prime}(0)$ differ only after desired significant digit. The last value of $\eta_{\infty}$ is taken as the finite value for determining the velocity, temperature and concentration, respectively. After getting all the initial conditions, we solve this system of simultaneous equations using fourth order Runge-Kutta integration scheme. The effects of the flow parameters on the velocity, temperature and nanoparticle concentration, are computed, discussed and have been graphically represented in figures and the local skin friction and rate of heat transfer are shown in Table 3 for various values of different parameters. Now for performing the present solution we have to define new variables by the equations $y_{1}=f, y_{2}=f^{\prime}, y_{3}=f^{\prime \prime}, y_{4}=\theta, y_{5}=\theta^{\prime}, y_{6}=\phi, y_{7}=\phi^{\prime}$. In this respect, we have chosen a step size of $\Delta \eta=0.002$ to satisfy the convergence criterion of $10^{-6}$ in all cases. The value of $\eta_{\infty}$ has been found to each iteration loop by $\eta_{\infty}=\eta_{\infty}+\Delta \eta$. The maximum value of $\eta_{\infty}$ for each group of parameters $M, \beta$, $\lambda, N b, N t, P r$ and $L e$ has been determined when the values of the unknown boundary conditions does not change to successful loop with an error less than $10^{-6}$.

\subsection{Code validation}

In the absence of magnetic parameter, Brownian motion and thermophoresis parameter, the present results are almost same with White (1991), Mohammadi et al. (2012) and Khan and Pop (2013) as displayed in Table 2. To check the validity of the present code, the values of $f^{\prime \prime}(0)$ has been calculated for $M=\lambda=N b=N t=P r=L e$ $=0$ and for different values of wedge angle parameter $\beta$. From the Table 2, it is observed that the data produced by the present code and those of mentioned author's shows excellent agreement between two sets of data and, so justifies the use of the present numerical code for current model. 
Table 2: Comparis on of skin friction [ $\mathrm{f}^{\prime \prime}(0)$ ] for different values of $\beta$, when $M=P r=N b=N t=L e=\lambda=0$

\begin{tabular}{ccccc}
\hline & $\begin{array}{c}\text { White } \\
(1991)\end{array}$ & $\begin{array}{c}\text { Mohammadi et al. } \\
(2012)\end{array}$ & $\begin{array}{c}\text { Khan and Pop } \\
(2013)\end{array}$ & $\begin{array}{c}\text { Present } \\
\text { results }\end{array}$ \\
\hline$\beta$ & $f^{\prime \prime}(0)$ & $f^{\prime \prime}(0)$ & $f^{\prime \prime}(0)$ & $f^{\prime \prime}(0)$ \\
\hline-0.12 & - & 0.281772 & - & 0.28211 \\
-0.15 & - & 0.216335 & - & 0.217153 \\
-0.18 & - & 0.128637 & - & 0.13138 \\
0.0 & 0.4696 & 0.469589 & 0.4696 & 0.46964 \\
0.2 & - & - & - & 0.686690 \\
$1 / 6$ & 0.6550 & - & 0.6550 & 0.6550 \\
$1 / 3$ & 0.8021 & - & 0.8021 & 0.80212 \\
0.5 & 0.9277 & 0.927601 & 0.9277 & 0.92768 \\
$2 / 3$ & 1.0389 & - & 1.0389 & 1.0389 \\
1.0 & 1.2326 & 1.232587 & 1.2326 & 1.232587 \\
1.6 & - & - & - & 1.5215139 \\
\hline
\end{tabular}

\section{Results and Discussion}

The nonlinear-coupled ordinary differential equations along with boundary conditions are solved numerically using Runge-Kutta $4^{\text {th }}$ order integration scheme with shooting method. The effect of various parameters on velocity, temperature and nanoparticle concentration has been shown graphically and in tabular form.

\subsection{Velocity field}

The effect of pressure gradient parameter, magnetic parameter and stretching ratio parameter on velocity profiles has been shown in Figs. 2-5. From Fig. 2, it is observed that the velocity profile increases with the increase in values of pressure gradient parameter $\beta$ in absence of entering parameters and the separation arises at $\beta=-0.198$ but in presence of stretching ratio parameter the flow separation are occurred at $\beta=-0.35$.

The effect of magnetic field parameter on velocity profile $f^{\prime}(\eta)$ is pictured in Fig. 4. The essence of the magnetic parameter has been explained from the sign of the term in Eq. (2). This term is composed of the imposed pressure force and the Lorentz force, which slows down the fluid motion in the boundary layer region. When the imposed pressure force overcomes the Lorentz force $(U>u)$, the effect of the magnetic parameter increases the velocity. Similarly, when the Lorentz force dominates the imposed pressure force $(u>U)$, the effect of the magnetic parameter decreases velocity flow and hence it decreases momentum boundary layer thickness. From Fig. 5, for a fixed value of $\eta$, as the parameter $\lambda$ increases, the velocity profile also increases and finally it is getting constant, as $\lambda$ is closer to one.

\subsection{Thermal field}

The temperature profiles depicts in Figs. 6-9. From these figures, it is seen that the temperature increases for the increase in thermophoresis parameter $N t$ but reverse results arise for Brownian motion parameter, stretching ratio parameter and Prandtl number. Increase in $N t$ causes increment in the thermophoretic force which tends to move nanoparticles from hot to cold areas and consequently it increases the magnitude of temperature. The increment of Prandtl number results in major effects on temperature profile. The thermal boundary layer thickness reduces with Prandtl number and it happens due to decrease of thermal diffusivity for the increment of Prandtl number. As a result, the heat transfer rate is increasing for Brownian motion parameter, stretching ratio parameter and Prandtl number but reverse trend arises in case of thermophoresis parameter. 


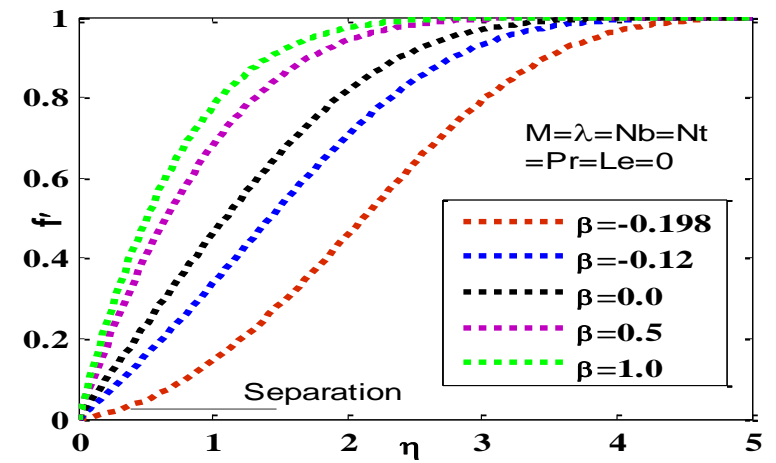

Fig. 2: Velocity profiles for various values of $\beta$

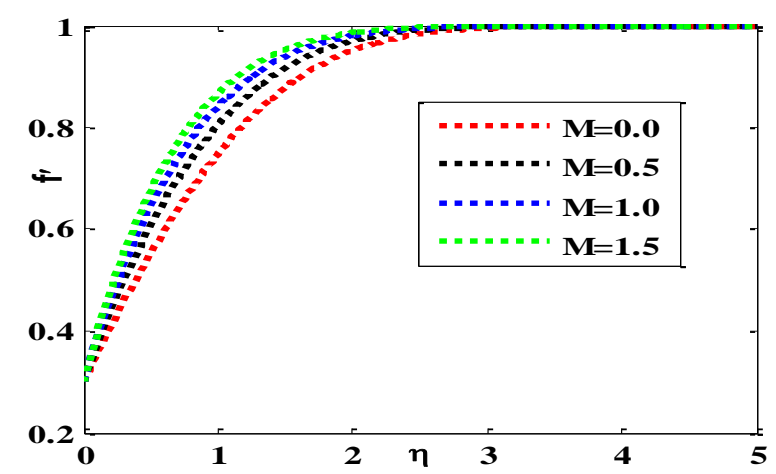

Fig. 4: Velocity profiles for various values of $\mathrm{M}$

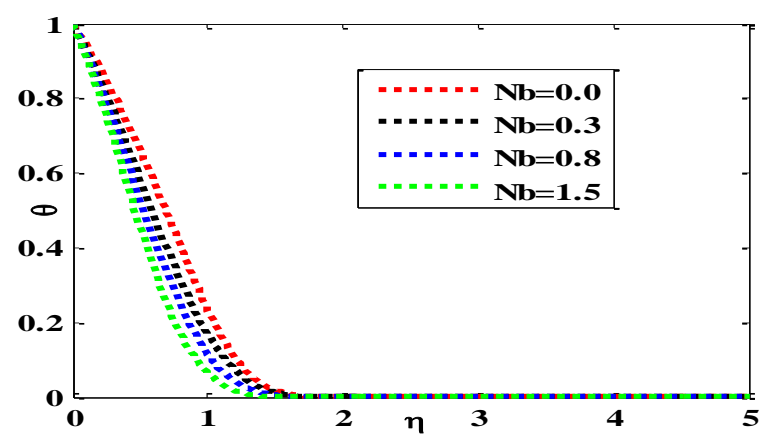

Fig. 6: Temperature profiles for $\mathrm{Nb}$

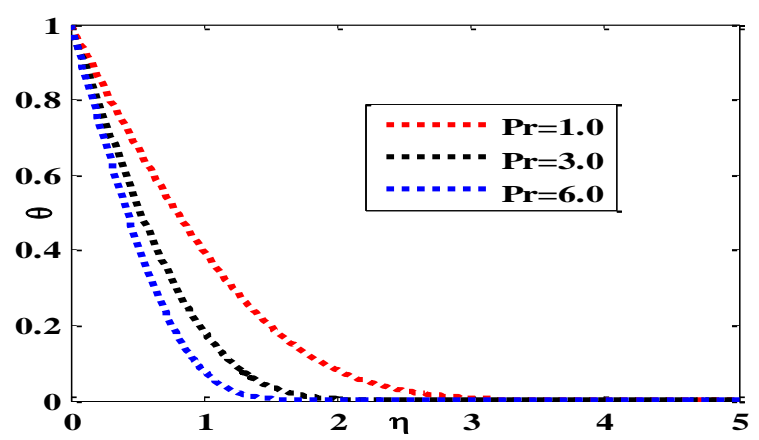

Fig. 8: Temperatures profiles for $\mathrm{Pr}$

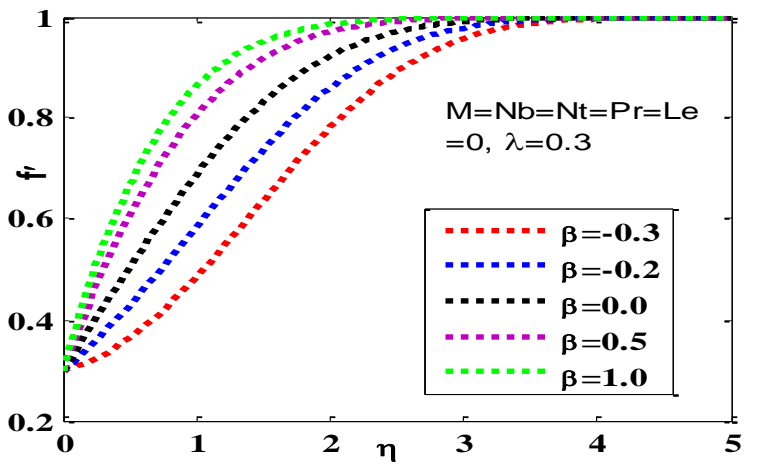

Fig. 3: Velocity profiles for $\beta$ with $\lambda=0.3$

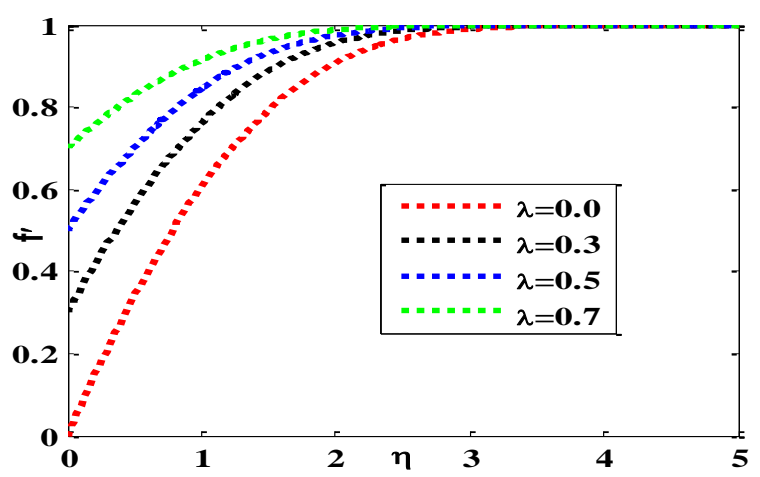

Fig. 5: Velocity profiles for various values of $\lambda$

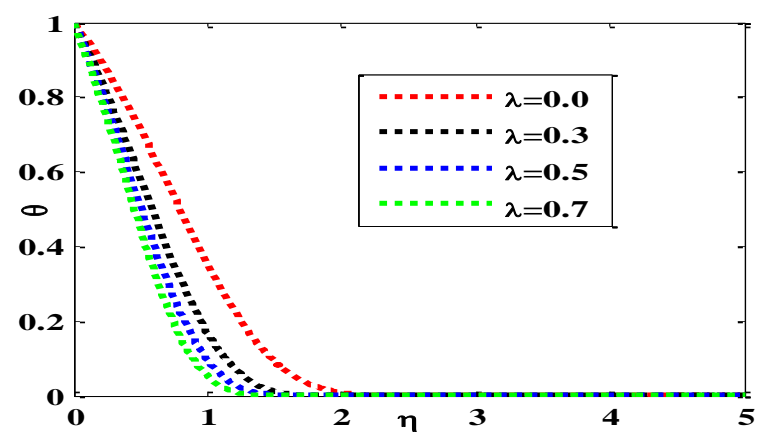

Fig. 7: Temperature profiles for $\lambda$

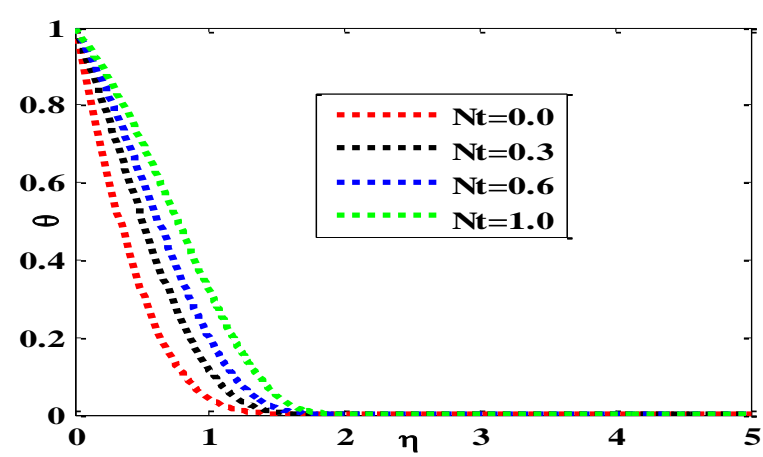

Fig. 9: Temperature profiles for $N t$ 


\subsection{Nanoparticle concentration}

The variation of nanoparticle concentration has been shown in Figs. 10-13. It is seen that the nanoparticle concentration increases for thermophoresis parameter $N t$ but reverse results arise for pressure gradient parameter, Brownian motion parameter and Lewis number. Thermophoresis parameter $N t$ is a key parameter for analyzing the temperature distributions and nanoparticles volume fraction in nanofluid flow. Therefore, increase of $N t$, the temperature profile and nanoparticle concentration of the fluid increases. Increasing $N t$ causes increment in the thermophoresis force which tends to move nanoparticles from hot to cold areas and consequently it increases the magnitude of temperature profiles and nanoparticle concentration profiles. Ultimately, the thickness of nanoparticle concentration boundary layer becomes significantly large for slightly increased value of thermophoresis parameter. Fig. 11 illustrates the effect of Lewis number on concentration profile. It is clearly shown in the figure that as the Lewis number increases, the concentration profile decreases significantly. This is because the increment of Lewis number reduces Brownian diffusion coefficient and this leads the flow to decline the concentration profile. This is obvious from the very definition of the parameter.

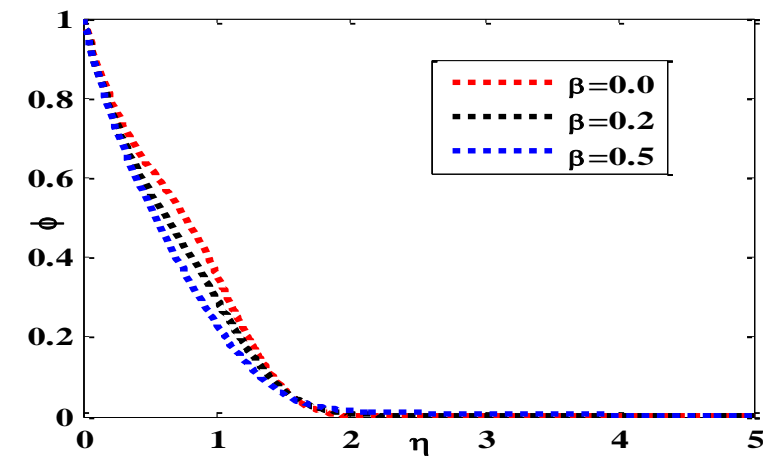

Fig. 10: Nanoparticle concentration for $\beta$

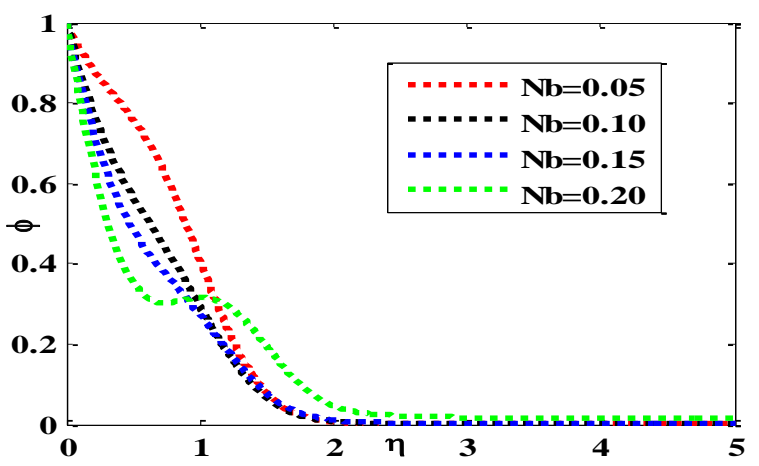

Fig. 12: Nanoparticle concentration for $\mathrm{Nb}$

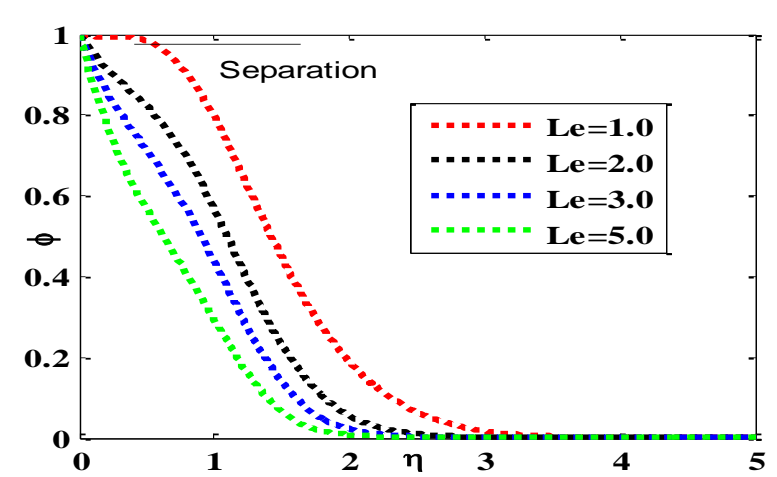

Fig. 11: Nanoparticle concentration for Le

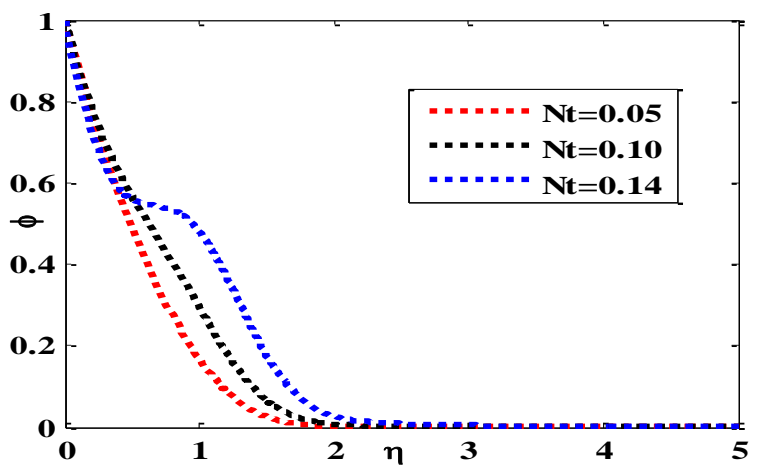

Fig. 13: Nanoparticle concentration for $N t$

Again, the various values of skin friction, rate of heat transfer and rate of nanoparticle concentration are presented in Table 3 for different values of $N b, N t, \lambda$ and $L e$ when $\operatorname{Pr}=1.0$ and $M=0.1$.

Table 3: Values of skin friction $\left[f^{\prime \prime}(0)\right]$, local Nusselt number $\left[-\theta^{\prime}(0)\right]$ and local Sherwood number $\left[-\varphi^{\prime}(0)\right]$ for different values of $N b, N t, \lambda$ when $\operatorname{Pr}=1.0$ and $M=0.1$.

\begin{tabular}{ccccccc}
\hline$\lambda$ & $N b$ & $N t$ & $L e$ & $f^{\prime \prime}(0)$ & $-\theta^{\prime}(0)$ & $-\varphi^{\prime}(0)$ \\
\hline $\mathbf{0 . 3}$ & 0.4 & 0.4 & 2.0 & 0.59710 & 0.64858 & 0.70207 \\
$\mathbf{0 . 5}$ & 0.4 & 0.4 & 2.0 & 0.45634 & 0.76658 & 0.78802 \\
$\mathbf{0 . 7}$ & 0.4 & 0.4 & 2.0 & 0.29051 & 0.87148 & 0.92169 \\
\hline
\end{tabular}


M. Ali, M. A. Alim, R. Nasrin, M. S. Alam/Journal of Naval Architecture and Marine Engineering, 14(2017)135-144

\begin{tabular}{lllllll}
\hline 0.3 & $\mathbf{0 . 8}$ & 0.4 & 2.0 & - & 0.71569 & 0.75069 \\
0.3 & $\mathbf{1 . 5}$ & 0.4 & 2.0 & - & 0.80455 & 0.78123 \\
0.3 & 0.4 & $\mathbf{0 . 3}$ & 2.0 & - & 0.83799 & 0.70722 \\
0.3 & 0.4 & $\mathbf{0 . 6}$ & 2.0 & - & 0.58416 & 0.74463 \\
0.3 & 0.4 & 0.4 & $\mathbf{1 . 0}$ & -- & - & 0.45265 \\
03 & 0.4 & 0.4 & $\mathbf{3 . 0}$ & - & - & 0.87037 \\
\hline
\end{tabular}

\section{Conclusions}

In this paper, the classical Falkner-Skan flow over a stretching wedge surface in a nanofluid in presence of magnetic field has been studied. From the present numerical calculation, the following conclusions can be made: The velocity field increases for the increase in values of pressure gradient parameter, magnetic parameter and stretching ratio parameter. As a result, the thickness of momentum boundary layer decreases. The temperature field decreases for the increase in values of Brownian motion, stretching ratio and Prandtl number but increases for the increase in thermophoresis parameter. The nanoparticle concentration field decreases for the increase in values of pressure gradient parameter, Brownian motion parameter and Lewis number whereas it increases for thermophoresis parameter. From Figs. 2 and 3, it is observed that the flow separation occurs at $\beta \geq-0.198$ and $\beta$ $\geq-0.3$ respectively.

\section{References}

Adekeye, T., Adegun, I., Okekunle P., Hussein, A. K., Oyedepo, S., Adetiba, E. and Fayomi, O. (2017): Numerical analysis of the effects of selected geometrical parameters and fluid properties on MHD natural convection flow in an inclined elliptic porous enclosure with localized heating, Heat Transfer-Asian Research, Vol. 46, pp. 261-293. https://doi.org/10.1002/htj.21211

Ahmed, S., Hussein, A. K., Mohammed, H., and Sivasankaran, S. (2014): Boundary layer flow and heat transfer due to permeable stretching tube in the presence of heat source/sink utilizing nanofluids, Applied Mathematics and Computation, Vol. 238, pp. 149-162. https://doi.org/10.1016/j.amc.2014.03.106

Ahmed, S., Hussein, A. K., Mohammed, H., Adegun, I., Zhang, X., Kolsi, L., Has anpour, A., and Sivasankaran, S. (2014): Viscous dissipation and radiation effects on MHD natural convection in a square enclosure filled with a porous medium, Nuclear Engineering and Design, Vol. 266, pp. 34-42.

https://doi.org/10.1016/j.nucengdes.2013.10.016

Ashwini, G, Eswara, A. T. (2015): Unsteady MHD decelerating flow past a wedge with internal heat generation / absorption, International Journal of Mathematics and Computational Science, Vol. 1, pp. 13-26.

Buongiorno, J. (2006): Convective transport in nanofluids, Journal of Heat Transfer, Vol. 128, pp. $240-250$.

https://doi.org/10.1115/1.2150834

Choi, S. U. S. (1995): Enhancing thermal conductivity of fluids with nanoparticles, International Mechanical Engineering Congress and Exposition, San Francisco, Vol. 66, USA, ASME, FED 231/MD, pp. 99-105.

Chand, R., Rana, G C. and Hussein, A. K. (2015): On the onset of thermal Instability in a low Prandtl number nanofluid layer in a porous medium, Journal of Applied Fluid Mechanics, Vol. 8, pp. 265-272.

https://doi.org/10.18869/acadpub.jafm.67.221.22830

Devi, M. B., Gangadhar, K. and Kumar P. S. (2017): Effect of viscous dissipation on power law - fluid past a permeable stretching sheet in a porous media, International Journal of Advanced Research in Computer Science, Vol. 8, pp. 113-118.

Falana, F., Ojewale, O. A. and Adeboje, T. B. (2016): Effect of Brownian motion and thermophoresis on a nonlinearly stretching permeable sheet in a nanofluid, Advances in Nanoparticles, Vol. 5, pp. 123-134.

https://doi.org/10.4236/anp.2016.51014

Haile, E. and Shankar, B. (2015): Boundary-layer flow of nanofluids over a moving surface in the presence of thermal radiation, viscous dissipation and chemical reaction, Applications and Applied Mathematics, Vol. 10, pp. 952-969. 
Hayat, T., Majid, H., Nadeem, S. and Meslou S., (2011): Falkner-Skan wedge flow of a power-law fluid with mixed convection and porous medium, Computers and Fluids, Vol. 49, pp. 22-28.

https://doi.org/10.1016/j.compfluid.2011.01.020

Hussein, A. K., Ashorynejad, H., Shikholeslami, M., and Sivasankaran, S. (2014): Lattice Boltzmann simulation of natural convection heat transfer in an open enclosure filled with $\mathrm{Cu}$-water nanofluid in a presence of magnetic field, Nuclear Engineering and Design, Vol. 268, pp. 10-17.

https://doi.org/10.1016/j.nucengdes.2013.11.072

Khan, W. A. and Pop, I. (2013): Boundary layer flow past a wedge moving in a nanofluid, Mathematical Problem and Engineering, Vol. 1, 7 pages.

Khan, U., Ahmed, N., Mohyud-Din, S. T. and Bin-Mohsin, B. (2017): Nonlinear radiation effects on MHD flow of nanofluid over a nonlinearly stretching/shrinking wedge, Neural Computing \& Applications, Vol. 28, pp. 2041-2050. https://doi.org/10.1007/s00521-016-2187-x

Kandasamy, R., and Mohamad, R. (2015): Radiative heat transfer on nanofluids flow over a porous convective surface in the presence of magnetic field, Journal of Applied Mechanical Engineering, Vol. 4, pp. 1-7.

Mohammadi, F., Hosseini, M. M, Dehgahn, A. and Maalek Ghaini F. M. (2012): Numerical solutions of Falkner-Skan equation with heat transfer, Studies in Nonlinear Science, Vol. 3, pp. 86-93.

Nasrin, R.(2011): Finite element simulation of hydromagnetic convective flow in an obstructed cavity, International Communications in Heat and Mass Transfer, Vol. 38, No. 5, pp. 625 - 632.

https://doi.org/10.1016/j.icheatmass transfer.2011.02.005

Nasrin, R. and Alim, M. A. (2012): Soret and Dufour effects on double diffusive natural convection in a chamber using nanofluid, International Journal of Heat \& Technology, Vol. 30, No. 1, pp. 111-120.

Nasrin, R. and Alim, M. A., (2012): Control volume finite element simulation of MHD forced and natural convection in a vertical channel with a heat-generating pipe, International Journal of Heat and Mass Transfer, Vol. 55, No. 11-12, pp. 2813-2821. https://doi.org/10.1016/j.ijheatmas stransfer.2012.02.023

Nasrin, R. and Alim, M. A., (2014): Semi-empirical relation for forced convective analysis through a solar collector, Solar Energy, Vol. 105, pp. 455-467. https://doi.org/10.1016/j.solener.2014.03.035

Nachtsheim, P. R. and Swigert, P., (1965): Satisfaction of the asymptotic boundary conditions in numerical solution of the systems of non-linear equations of boundary layer type, Ph.D. Thesis, NASA TN D- 3004, Washington, D.C.

Parvin, S., Nasrin, R., Alim, M. A. and Hossain, M. A. (2012): Double diffusive natural convection in a partially heated enclosure using nanofluid, Heat Transfer-Asian Research, Vol. 41, No. 6, pp. 484-497.

https://doi.org/10.1002/htj.21010

Shaw, S., Kameswaran, P. K. and Sibanda, P. (2016): Effects of slip on nonlinear convection in nanofluid flow on stretching surfaces, Boundary value Problems, Vol. 2016. https://doi.org/10.1186/s 13661-015-0506-2

Srinivasacharya, D., Mendu, U. and Venumadhav, K. (2015): MHD boundary layer flow of a nanofluid past a wedge, Procedia Engineering, Vol. 127, pp.1064 - 1070. https://doi.org/10.1016/j.proeng.2015.11.463

Ullah, I., Khan, I. and Shafie, S. (2017): Heat and mass transfer in unsteady MHD slip flow of Casson fluid over a moving wedge embedded in a porous medium in the presence of chemical reaction: Numerical solutions using Keller-Box method, Numerical Methods for Partial Differential Equations, Vol. 2017, pp. 1-25.

https://doi.org/10.1002/num.22221

White, F. M. (1991): Viscous Fluid Flow, 2nd edn., McGraw-Hill, New York, NY, USA.

Yousif, M. A., Mahmood, B. A. and Rashidi M. M. (2017): Thermal boundary layer analysis of nanofluid flow over a stretching flat plate in different transpiration conditions by using DTM-Pad'e method, Journal of Mathematics and Computer Science, Vol. 17, pp. 84-95. https://doi.org/10.22436/jmcs.017.01.08

Yacob, A. N., Ishak, A. and Pop, I. (2011): Falkner-Skan problem for a static or moving wedge in nanofluids, International Journal of Thermal Science, Vol. 50, pp. 133-139.

https://doi.org/10.1016/j.ijthermalsci.2010.10.008 